\title{
THE ALTERNATION OF NODES OF LINEARLY \\ INDEPENDENT SOLUTIONS OF SECOND ORDER DIFFERENCE EQUATIONS*
}

\author{
BY OTTO DUNKEL
}

We shall consider solutions of the difference equation

$$
u(n+2)=A(n) u(n+1)-B(n) u(n), \quad B(n)>0,
$$

where $A(n)$ and $B(n)$ are finite and single-valued functions of the integer $n$. If the points obtained by plotting a solution $u(n)$ are joined by segments of a straight line, this broken line gives a representation of a single-valued and continuous function $f(x)$ such that $f(n)=u(n)$. The zeros of $f(x)$ are called the nodes of $u(n)$.

Proofs have already been given of the following theorem.

THEOREM. The nodes of two linearly independent solutions of (1) separate one another. $\dagger$

The proof which is to be given here seems simpler and more obvious than either of these two proofs. Two known and easily verified facts will be used. If $u_{1}(n)$ and $u_{2}(n)$ are any two solutions of (1) and if we set

then

$$
\Delta(n)=\left|\begin{array}{ll}
u_{1}(n) & u_{2}(n) \\
u_{1}(n+1) & u_{2}(n+1)
\end{array}\right|,
$$

$$
\Delta(n+1)=B(n) \Delta(n) .
$$

As a first result of the condition imposed upon $B(n)$ in (1) we have the fact that if $\Delta(n)$ is not zero for one value of $n$ then it is never zero and its sign remains unchanged.

A necessary and sufficient condition that the two solutions $u_{1}(n)$ and $u_{2}(n)$ are linearly independent is that $\Delta(n)$ is not zero for one value of $n$.

* Presented to the Society, December 29, 1925

$\dagger$ Porter, Annals of Mathematics, (2), vol. 3, (1901-02), p. 65. Moulton, E. J., ibid. ,(2), vol. 13 (1911-12), p. 137. 
We have

$f_{i}(x)=(x-n)\left[u_{i}(n+1)-u_{i}(n)\right]+u_{i}(n), \quad n \leqq x<n+1, \quad i=1,2$.

An easy calculation shows that

$$
W(x)=\left|\begin{array}{ll}
f_{1}(x) & f_{2}(x) \\
f_{1}^{\prime}(x) & f_{2}^{\prime}(x)
\end{array}\right|=\Delta(n), \quad n<x<n+1 .
$$

If we set

$$
f_{i}^{\prime}(n)=u_{i}(n+1)-u_{i}(n)
$$

then $W(x)$ is defined for all values of $x$, and it is never zero and it has always the same sign, if, as we shall now suppose, $u_{1}(n)$ and $u_{2}(n)$ are linearly independent.

We shall now have to notice a second result of the condition placed upon $B(n)$. If $u(n+1)=0, u(n+2)=-B(n) u(n)$, and hence $f^{\prime}(x)$, which may be discontinuous at $n+1$, has the same sign a little before, at, and a little after $n+1$. The case in which $u(n)$ is zero for two successive values of $n$ cannot occur here for two linearly independent solutions.

Suppose that $f_{1}(x)$ vanishes at $x_{1}$ and at $x_{2}$ but at no point between. Then

$$
f_{1}^{\prime}\left(x_{1}\right) f_{1}^{\prime}\left(x_{2}\right)<0,
$$

even if one or both of $x_{1}$ and $x_{2}$ are integers as a result of the remark above. Then from the facts stated above

$$
W\left(x_{1}\right) W\left(x_{2}\right)=f_{1}^{\prime}\left(x_{1}\right) f_{2}\left(x_{1}\right) f_{1}^{\prime}\left(x_{2}\right) f_{2}\left(x_{2}\right)>0 .
$$

From (4) and (5) it follows that

$$
f_{2}\left(x_{1}\right) f_{2}\left(x_{2}\right)<0,
$$

and this requires that $f_{2}(x)$, which is continuous, shall vanish at least once between $x_{1}$ and $x_{2}$. It cannot vanish more than once, for if it did then the same form of proof would show that $f_{1}(x)$ vanishes between $x_{1}$ and $x_{2}$ contrary to the hypothesis. This concludes the proof.

WASHINGTON UNIVERSITY 